\title{
Circulating miRNAs from imprinted genomic regions are associated with peripheral muscle strength in COPD patients
}

To the Editor:

Reduced muscle mass and strength are common comorbidities of COPD that lead to a poorer quality of life and are associated with increased mortality. Whilst the presence of disease may be viewed as a 'necessary' factor for the loss of muscle mass and strength, disease severity itself is not tightly linked to muscle loss, as some patients with mild disease have a significant loss of muscle mass and strength, whereas others with severe disease appear to maintain a normal muscle mass and strength [1]. These differences in susceptibility to changes in muscle are likely to be caused by the interplay between genetic, epigenetic and environmental factors.

Genetic contributors to muscle mass and strength in COPD include polymorphisms in the vitamin D receptor [2], angiotensin-converting enzyme [3] and the FTO gene [4]. Environmental factors include those directly associated with the pathophysiology of the disease (e.g. oxidative stress and inflammation [5]), consequences of disease (e.g. reduced physical activity $[5,6]$ ) as well as factors associated with the history of the individual (e.g. smoking history and developmental factors such as pre-natal growth [7]).

Mechanistically both cigarette smoking [8] and birth weight [9] are associated with differences in DNA methylation, a process normally associated with gene silencing. Polymorphic variation can also contribute to alterations in DNA methylation and thereby affect physical performance. For example, polymorphisms that reduce the activity of enzymes involved in methylation are enriched in elite athletes [10]. DNA methylation also contributes to the control of pre-natal growth in a process known as imprinting, where either the maternal or paternal allele becomes methylated and thereby, inactive [11]. Many paternally expressed genes are associated with promoting growth (e.g. IGF-2) whereas many maternally expressed genes are associated with limiting growth (e.g. IGF2R). We found that expression of paternally expressed miRNAs from a cluster on chromosome 19 (C19MC) was positively associated with fat free mass index (FFMI) in COPD patients whereas expression of the maternally expressed miR-675 was inversely proportional to FFMI and strength [12]. Furthermore, methylation of the imprinting control region associated with miR-675 expression was higher in weak patients than in those with normal strength [12]. However, there was no association of miR-675 or C19MC miRNAs with muscle phenotype in healthy elderly people suggesting that the relative expression levels of the imprinted miRNAs have physiological consequences only in the presence of disease.

As miRNAs are present in circulation we hypothesised that differences in miRNAs would be present in the plasma of patients with low and normal FFMIs and that the levels of these miRNAs would be associated with strength and therefore screened for miRNAs that were differentially present in the circulation of GOLD 3-4 male COPD patients with a low FFMI $(n=7)$ compared to those with a normal FFMI $(n=7)$. Six of the 13 miRNAs different at $\mathrm{p}<0.05$ were derived from a single imprinted miRNA cluster located on chromosome 14 (C14MC) and included both the $3 p$ and $5 p$ forms of miR- 485 .

Analysis of plasma miR-485-3p levels in a larger group of patients of both sexes and all GOLD stages $(n=70)$ did not confirm the difference between circulating miR-485-3p in patients with a low or normal FFMI (figure 1a) nor show an association of miR-485-3p with FFMI in the group as a whole or in the

@ERSpublications

Circulating levels of miR-485-3p, a maternally expressed miRNA, are associated with muscle strength in COPD patients http://ow.ly/Wq51309fQtK

Cite this article as: Lee JY, Donaldson AV, Lewis A, et al. Circulating miRNAs from imprinted genomic regions are associated with peripheral muscle strength in COPD patients. Eur Respir J 2017; 49: 1601881 [https://doi.org/10.1183/13993003.01881-2016]. 

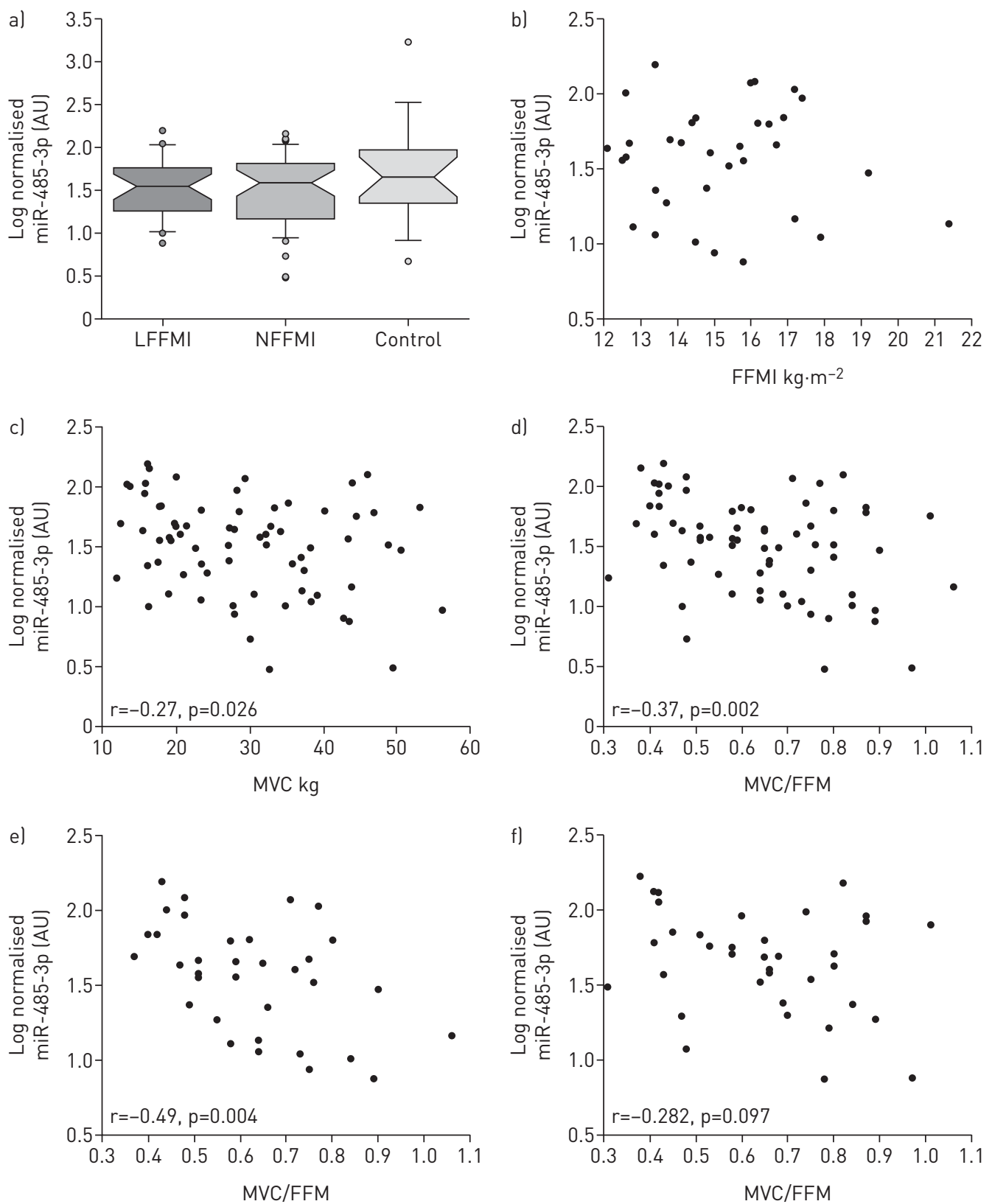

FIGURE 1 Association of miR-485-3p with clinical parameters in COPD patients. Circulating levels of miR-485-3p were determined in patients with COPD and controls. Levels of miR-485-3p were not different between patients and controls or between patients with a normal (N) or low (L) fat free mass index (FFMI) (a), nor was miR-485-3p associated with FFMI in patients with GOLD 3-4 COPD (b). Circulating miR-485-3p levels were inversely proportional to maximal voluntary contraction in GOLD1-4 COPD patients (c), and this association was stronger when strength was normalised to fat free mass (d). Subgroup analysis showed that the association was stronger in patients with severe (GOLD 3-4) COPD (e) than in patients with mild (GOLD 1-2) COPD ( $f$ ). MVC: maximum voluntary contraction.

subset of patients with the same disease severity (GOLD 3-4, figure 1b). However, miR-485-3p was associated with strength (maximum voluntary contraction, MVC) in the whole cohort of patients $(r=-0.27$, $\mathrm{p}=0.026$, figure 1c). Furthermore, miR-485-3p was more tightly associated with strength when normalised for FFM ( $\mathrm{r}=-0.37, \mathrm{p}=0.002$, figure $1 \mathrm{~d}$ ) suggesting either muscle quality or lower limb mass contributed to the association. Such a rationale could also explain the association with muscle strength but not FFMI.

Subgroup analysis showed that whilst there was no difference in the expression of miR-485-3p between COPD patients and controls (figure 1a) nor any association with lung function measured as FEV1, or $\mathrm{TL}_{\mathrm{CO}}$, the association of miRNA levels with muscle strength was only present in patients with severe 
disease (GOLD 3-4) and not in those with milder disease ( $\mathrm{r}=-0.49 \mathrm{p}=0.004, \mathrm{n}=35$ and $\mathrm{r}=-0.28, \mathrm{p}=0.097$ $\mathrm{n}=36$ respectively figure $1 \mathrm{e}$ and $\mathrm{f}$ ).

Comparison of circulating miR-485-3p levels with the expression of H19 (another maternally expressed gene) in muscle showed a significant association $(\mathrm{r}=-0.37, \mathrm{p}=0.013)$.

We have previously measured circulating levels of myomiRs (miRNAs enriched in muscle) in these same patients and found that miR-1 expression was associated with strength [13]. Comparison of circulating miR-485-3p levels with those of the muscle derived miRNAs showed that circulating miR-485-3p was inversely proportional to levels of miR-499, miR-206 and miR-133 ( $r=-0.63, p<0.001, r=-0.60, p<0.001$, $\mathrm{r}=-0.63, \mathrm{p}<0.001)$.

Our data show that circulating levels of the maternally expressed miRNA miR-485-3p reflect the expression of a maternally expressed mRNA in muscle and are inversely associated with muscle strength in patients, most notably when normalised to FFM. The observation that the levels of miR-485-3p are not different between patients and controls, and that the associations are strongest in those with the most severe disease implies (a) that disease does not significantly alter miR-485-3p levels in circulation and (b) that those at the high end of the normal distribution of miR-485-3p expression are more likely to lose muscle strength. As maternally expressed genes tend to be associated with reduced growth and in particular reduced stem cell proliferation, our data suggest that individuals with a low intrinsic rate of regeneration may be most susceptible to losing muscle strength in the presence of disease. This observation is consistent with previous observations of reduced centralised nuclei in COPD patients with the lowest FFMI $[12,14]$ and with the positive association of paternally expressed miRNAs from the C19MC cluster with FFMI in COPD patients. In that study, we also showed that the expression of imprinted miRNAs was not associated with muscle mass in normal healthy individuals indicating that it is only under conditions of physiological stress that the effects of these imprinted genes become apparent.

Circulating levels of miR-485-3p were negatively associated with those known to be derived from muscle. Consequently, muscle does not appear to be the major source of circulating miR-485-3p. We therefore propose that this maternally expressed miRNA acts as a marker of the potential for regeneration. Any effect of miR-485-3p on muscle wasting is likely to be through effects on the proliferation of satellite cells and consistent with this suggestion, both miR-485-3p and miR-485-5p have been shown to inhibit cell proliferation in other cells [15]. This observation does, however, suggest that differences between patients who lose muscle mass and those who maintain muscle mass are detectable and raise the potential for a minimally invasive method to identify individuals at higher risk of losing muscle strength in response to the physiological stress imposed by COPD. Part of such a test may include the measurement of miR-485-3p, although the current data show that on its own this is not sufficient. Such a method may help to identify individuals at risk of muscle loss or identify those who would be suitable for inclusion in trials for anabolic agents.

Jen Y. Lee ${ }^{1}$, Anna V. Donaldson ${ }^{2}$, Amy Lewis ${ }^{1}$, S. Amanda Natanek ${ }^{1}$, Michael I. Polkey ${ }^{2}$ and Paul R. Kemp $\circledast^{1}$ ${ }^{1}$ National Heart and Lung Institute, Imperial College London, London, UK. ${ }^{2}$ National Institute for Health Research Respiratory Biomedical Research Unit at Royal Brompton and Harefield NHS Foundation Trust and Imperial College London, London, UK.

Correspondence: Paul R. Kemp, National Heart and Lung Institute, Imperial College London, Sir Alexander Fleming Building, South Kensington Campus, London, SW7 2AZ. E-mail: p.kemp@imperial.ac.uk

Received: Sept 232016 | Accepted after revision: Jan 232017

Support statement: This work was supported by the MRC COPDMAP and the NIHR Respiratory Disease Biomedical Research Unit at the Royal Brompton and Harefield NHS Foundation Trust and Imperial College London who part funded M.I. Polkey's salary and wholly funded A.V. Donaldson. Funding information for this article has been deposited with the Crossref Funder Registry.

Conflict of interest: Disclosures can be found alongside this article at erj.ersjournals.com

Acknowledgements: The views expressed in this publication are those of the authors and not necessarily those of the NHS, The National Institute for Health Research or the Department of Health.

\section{References}

1 Shrikrishna D, Patel M, Tanner RJ, et al. Quadriceps wasting and physical inactivity in patients with COPD. Eur Respir J 2012; 40: 1115-1122.

2 Hopkinson NS, Li KW, Kehoe A, et al. Vitamin D receptor genotypes influence quadriceps strength in chronic obstructive pulmonary disease. Am J Clin Nutr 2008; 87: 385-390.

3 Hopkinson NS, Nickol AH, Payne J, et al. Angiotensin converting enzyme genotype and strength in chronic obstructive pulmonary disease. Am J Respir Crit Care Med 2004; 170: 395-399. 
4 Wan ES, Cho $\mathrm{MH}$, Boutaoui $\mathrm{N}$, et al. Genome-wide association analysis of body mass in chronic obstructive pulmonary disease. Am J Respir Cell Mol Biol 2011; 45: 304-310.

5 Man WD, Kemp P, Moxham J, et al. Skeletal muscle dysfunction in COPD: clinical and laboratory observations. Clin Sci 2009; 117: 251-264.

6 Waschki B, Kirsten AM, Holz O, et al. Disease progression and changes in physical activity in patients with chronic obstructive pulmonary disease. Am J Respir Crit Care Med 2015; 192: 295-306.

7 Shaheen S. The beginnings of chronic airflow obstruction. Br Med Bull 1997; 53: 58-70.

8 Vucic EA, Chari R, Thu KL, et al. DNA methylation is globally disrupted and associated with expression changes in chronic obstructive pulmonary disease small airways. Am J Respir Cell Mol Biol 2014; 50: 912-922.

9 Jacobsen SC, Gillberg L, Bork-Jensen J, et al. Young men with low birthweight exhibit decreased plasticity of genome-wide muscle DNA methylation by high-fat overfeeding. Diabetologia 2014; 57: 1154-1158.

10 Terruzzi I, Senesi P, Montesano A, et al. Genetic polymorphisms of the enzymes involved in DNA methylation and synthesis in elite athletes. Physiol Genomics 2011; 43: 965-973.

11 Delaval K, Feil R. Epigenetic regulation of mammalian genomic imprinting. Curr Opin Genet Dev 2004; 14: 188-195.

12 Lewis A, Lee JY, Donaldson AV, et al. Increased expression of H19/miR-675 is associated with a low fat free mass index in patients with COPD. J Sarcopenia Cachexia Muscle 2016; 7: 330-344.

13 Donaldson A, Natanek SA, Lewis A, et al. Increased skeletal muscle-specific microRNA in the blood of patients with COPD. Thorax 2013; 68: 1140-1149.

14 Thériault ME, Paré MÈ, Maltais F, et al. Satellite cells senescence in limb muscle of severe patients with COPD. PLoS One 2012; 7: e39124.

15 Lou C, Xiao M, Cheng S, et al. miR-485-3p and miR-485-5p suppress breast cancer cell metastasis by inhibiting PGC-1alpha expression. Cell Death Dis 2016; 7: e2159. 